\title{
Observations of Magnetic Reconnection in the Transition Region of Quasi-Parallel Shocks
}

I. Gingell ${ }^{1}$, S.J. Schwartz ${ }^{2,1}$, J. P. Eastwood ${ }^{1}$, J. L. Burch ${ }^{3}$, R. E. $\operatorname{Ergun}^{2}$, S. Fuselier $^{3}$, D. J. Gershman ${ }^{4}$, B. L. Giles ${ }^{4}$, Y. V. Khotyaintsev ${ }^{5}$, B. Lavraud ${ }^{6}$, P.-A. Lindqvist ${ }^{5}$, W. R. Paterson ${ }^{4}$, T. D. Phan $^{7}$, C. T. Russell ${ }^{8}$, J. E. Stawarz $^{1}$, R. J. Strangeway ${ }^{8}$, R. B. Torbert ${ }^{9}$, F. Wilder ${ }^{2}$

${ }^{1}$ The Blackett Laboratory, Imperial College London, SW7 2AZ, United Kingdom

${ }^{2}$ Laboratory for Atmospheric and Space Physics, University of Colorado, Boulder, Colorado 80303, USA

${ }^{3}$ Southwest Research Institute, San Antonio, Texas 78238, USA

${ }^{4}$ NASA, Goddard Space Flight Center, Greenbelt, Maryland 20771, USA

${ }^{5}$ Swedish Institute of Space Physics (Uppsala), Uppsala, Sweden

${ }^{6}$ Institut de Recherche en Astrophysique et Planétologie, CNRS, UPS, CNES, Université de Toulouse,

Toulouse, France

${ }^{7}$ Space Science Laboratory,University of California, Berkeley, California, USA

${ }^{8}$ University of California, Los Angeles, Los Angeles, California 90095, USA

${ }^{9}$ University of New Hampshire, Durham, New Hampshire 03824, USA

\section{Key Points:}

- Reconnecting current sheets have been observed at a quasi-parallel bow shock.

- The ion-scale current sheet exhibits only an electron jet and heating, with no ion response.

- Consistent with kinetic simulations, reconnection relaxes complexity in the shock transition region.

Corresponding author: Imogen Gingell, i.gingell@imperial.ac.uk 


\begin{abstract}
Using observations of Earth's bow shock by the Magnetospheric Multiscale mission, we show for the first time that active magnetic reconnection is occurring at current sheets embedded within the quasi-parallel shock's transition layer. We observe an electron jet and heating but no ion response, suggesting we have observed an electron-only mode. The lack of ion response is consistent with simulations showing reconnection onset on sub-ion timescales. We also discuss the impact of electron heating in shocks via reconnection.
\end{abstract}

\title{
1 Introduction
}

Collisionless shocks are found in many astrophysical plasma environments, including planetary and stellar bow shocks, interplanetary shocks in the solar wind, and supernova remnants (Burgess \& Scholer, 2015). In order to reduce flows from super- to subsonic speeds, collisionless shocks must dissipate energy by particle processes, i.e. they are by necessity kinetic plasma structures. Understanding these microphysical processes is critical for understanding particle heating and acceleration (Auer, Hurwitz, \& Kilb, 1962; Gosling \& Robson, 1985; Morse, Destler, \& Auer, 1972). The family of kinetic plasma processes responsible for energy dissipation is strongly dependent on shock parameters such as the Mach number, plasma beta, and the angle, $\theta_{B n}$, between upstream magnetic field and shock normal (Burgess \& Scholer, 2015).

In examining the non-stationary structure of quasi-parallel shocks $\left(\theta_{B n}<45^{\circ}\right)$, recent simulations have shown that processes within the shock foot can generate current sheets and magnetic islands (Gingell et al., 2017). The evolution of these regions is modulated by cyclic self-reformation of the shock ramp over ion time scales. Reformation is a kinetic process driven by ions reflected from the shock ramp (Biskamp \& Welter, 1972; Hada, Oonishi, Lembège, \& Savoini, 2003; Scholer, Shinohara, \& Matsukiyo, 2003), or by instabilities associated with whistler waves localised in the foot region (Scholer \& Burgess, 2007), or by instabilities of the backstreaming ions in the foreshock (Burgess, 1989, 1995; Krauss-Varban \& Omidi, 1991). Within the shock transition region, distinct from the magnetosheath downstream, magnetic islands merge to form larger scale structures that are convected towards the magnetopause. An example snapshot of one such simulation, revealing embedded current sheets and magnetic islands (twisted fields or flux ropes), is visible in Figure 1. Within this model, self-reformation and other foot instabilities gen- 
erate a region of disordered or turbulent magnetic fluctuations close to the shock ramp. Decay of these disordered fluctuations may then occur via magnetic reconnection at current sheets and magnetic islands. These structures and processes thus are closely associated with magnetic reconnection.

In this letter, we demonstrate for the first time that active magnetic reconnection is occurring in the transition region of Earth's quasi-parallel bow shock. We show that reconnecting current sheets are present within a disordered transition region close to the shock ramp, which is consistent with the appearance of these structures in recent hybrid and kinetic shock simulations (Bohdan, Niemiec, Kobzar, \& Pohl, 2017; Gingell et al., 2017; Matsumoto, Amano, Kato, \& Hoshino, 2015). Magnetic reconnection, for which localised changes in magnetic topology result in rapid transfer of energy from fields to particles, has been observed in detail by Magnetospheric Multiscale (MMS) at Earth's magnetopause (Burch et al., 2016) and more recently in the turbulent magnetosheath (Phan et al., 2018). In contrast to magnetosheath observations reported by Phan et al. (2018) and global hybrid simulations by Karimabadi et al. (2014), structures discused here appear within seconds of crossing the bow shock, suggesting a close association with shock processes and a rapid evolution. In the standard model, reconnection occurs within an electron-scale diffusion region (Burch et al., 2016; Vasyliunas, 1975), while at ion scales coupled ions are ejected from the diffusion region as bi-directional jets (Gosling, Skoug, McComas, \& Smith, 2005; Paschmann et al., 1979; Phan et al., 2000). Reconnection exhausts then extend to much larger scales. In turbulent plasmas, magnetic reconnection is thought to play an important role in dissipation of energy at kinetic scales (Matthaeus \& Lamkin, 1986; Retinò et al., 2007; Servidio, Matthaeus, Shay, Cassak, \& Dmitruk, 2009; Sundkvist, Retinò, Vaivads, \& Bale, 2007). Given the observations of electron heating detailed in this letter, we raise the question of how reconnection can contribute to shock energetics.

\section{Case Study of a Quasi-parallel Shock}

Here we discuss a crossing of Earth's bow shock by the four MMS spacecraft on 26 January 2017, 08:13:04 UTC. The mean spacecraft separation was 7km. Electromagnetic field data are provided by the flux gate magnetometer (FGM) (Russell et al., 2016) and electric field double probe (EDP), both within the FIELDS suite (Torbert et al., 2016). Particle data have been provided by the Fast Plasma Investigation (FPI) (Pollock et al., 


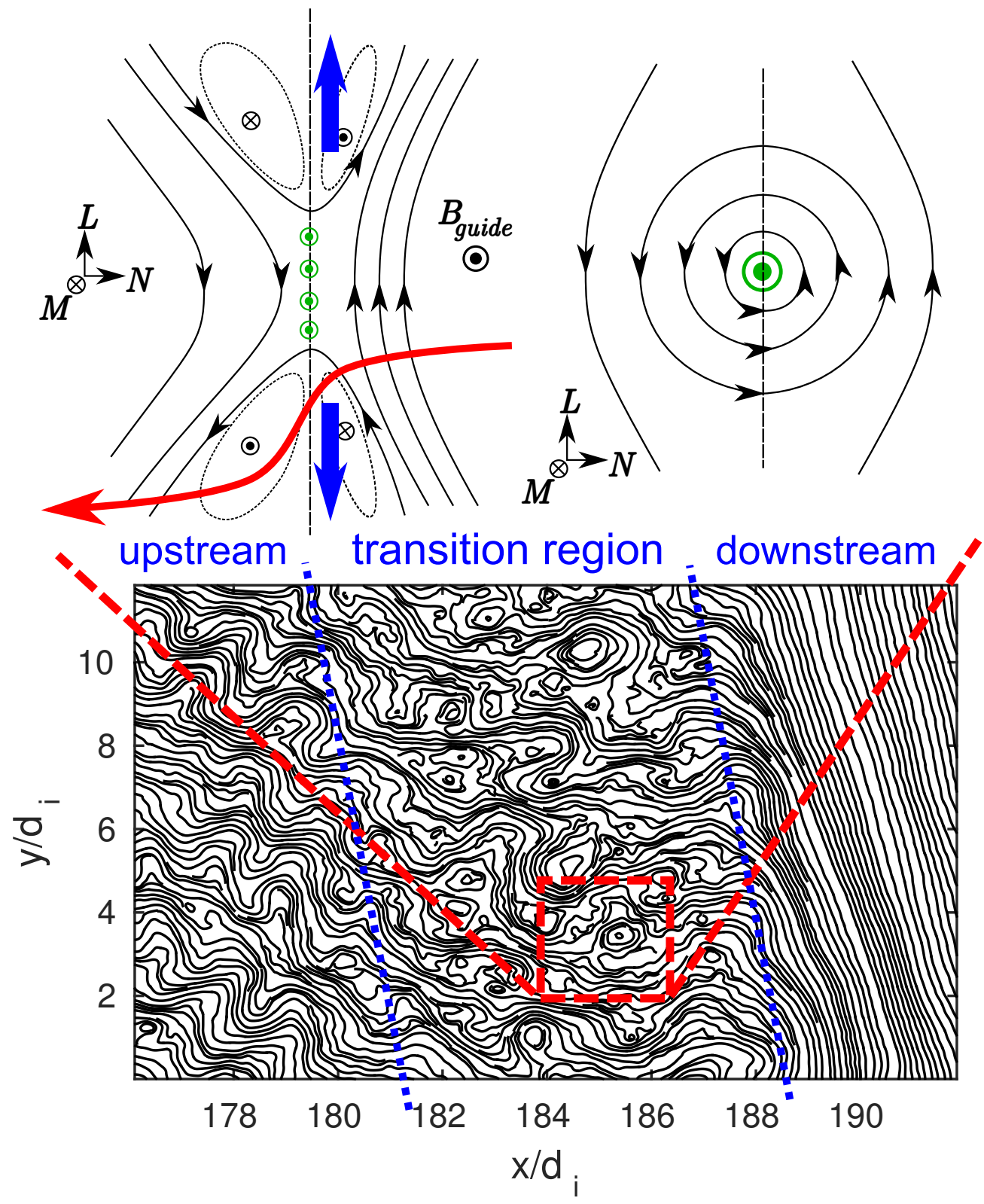

Figure 1. Top: Schematics of the magnetic structure (black), out-flowing jet directions (blue) and current densities (green) for an asymmetric, reconnecting current sheet (left) and rope-like twisted field structures (right). A red arrow depicts the trajectory of MMS1 through the structure observed in Figure 3. Bottom: Snapshot of the magnetic field line structure of a hybrid simulation of a reforming quasi-parallel shock (Gingell et al., 2017), demonstrating the appearance of current sheets and twisted field structures within the transition region. 
2016). The sampling frequency is $128 \mathrm{~Hz}$ for the FGM magnetic fields, and $8 \mathrm{kHz}$ for the EDP electric fields. The full three-dimensional ion phase space is sampled by FPI every $0.15 \mathrm{~s}$, and the electron phase space is sampled every $0.03 \mathrm{~s}$.

For the chosen event, the angle between the upstream magnetic field and shock normal is given by $\theta_{B n}=21^{\circ}$, the Alfvénic Mach number of the upstream flow is $M_{A}=$ 3 , the fast magnetosonic Mach number is $M_{\text {fast }} \approx 2$, and the upstream plasma beta is $\beta=1.4$. The Mach numbers and $\beta$ are determined from the mean fields and particle moments given by MMS for the upstream burst period 08:16:30 to 08:17:04 UTC, and downstream burst period 08:13:04 to 08:14:04. The shock normal $\hat{n}$ and hence the angle $\theta_{B n}$ is determined by mixed method, using the magnetic field and electron bulk velocities upstream and downstream of the shock (Abraham-Shrauner, 1972; Schwartz, 1998). Given the disordered and nonstationary nature of the shock transition layer for quasiparallel shocks, multiple spacecraft timing analysis is not reliable for determining the largescale orientation of the shock.

An overview of the event is shown for MMS1 in Figure 2. The magnetic field data in panel (a) demonstrates the presence of a transition region (highlighted in grey) between the relatively quiescent magnetosheath (before 08:14:04) and solar wind (after 08:16:04). Within this region the magnetic field is disordered, exhibiting multiple directional discontinuities. Using all four MMS spacecraft, we can use the curlometer method (Robert, Dunlop, Roux, \& Chanteur, 1998) to determine the barycentric current density, shown in panel (b). The high amplitude, narrow peaks within the current density (i.e. $\nabla \times B / \mu_{0}$ ) reveal several narrow current sheet-like structures with peak current densities on the order of $1 \mu \mathrm{Am}^{-2}$. This transition region is associated with significant fluctuations of the electron velocity, and enhancements in the electron number density and temperatures. Although we also observe fluctuations in the ion temperatures, there is no enhancement across the full transition region. We note that the change in field and plasma properties from the magnetosheath to the transition region at 08:14:04 may be in part associated with changes in the upstream plasma conditions rather than stationary shock structure.

In the solar wind, periodic reductions in the wind speed, visible at 08:16:20 and 08:16:40 in the ion differential energy flux and the bulk velocity $V_{e X}$ (panels $(\mathrm{g})$ and (c)), suggest that, as with the simulation in Figure 1, this shock may be undergoing cyclic self- 
reformation (Burgess, 1989) on a 20s timescale. Thus, this event is appropriate for evaluating the predictions of recent hybrid simulations of reforming, quasi-parallel shocks with respect to reconnection (see Gingell et al. (2017) and Figure 1).

\section{Current Sheets}

For discussion of individual coherent structures, we introduce a new coordinate system derived by using a hybrid minimum variance analysis (Gosling \& Phan, 2013; Phan et al., 2018). The current sheet normal $N$ is determined using $\mathbf{B}_{\mathbf{1}} \times \mathbf{B}_{\mathbf{2}} /\left|\mathbf{B}_{\mathbf{1}} \times \mathbf{B}_{\mathbf{2}}\right|$, where $\mathbf{B}_{1,2}$ are the fields at the two edges of the current sheet. The $M$ direction, corresponding to the current carrying direction, is given by $\mathbf{M}=\mathbf{L}^{\prime} \times \mathbf{N}$, where $\mathbf{L}^{\prime}$ is the direction of the maximum variance of the magnetic field. Finally, $\mathbf{L}=\mathbf{N} \times \mathbf{M}$.

Although many magnetic directional discontinuities are visible within the transition region shaded in Figure 2, we must observe electron or ion jets in order to conclude that these current sheets are actively reconnecting. These jets, corresponding to outflow of plasma from an active reconnection site, are expected in the $L$-direction. Structures in bulk velocity may be unipolar if the spacecraft crosses only one jet, or bipolar if the spacecraft crosses both jets. A schematic of the magnetic field, current and jet directions is shown in the top left of Figure 1.

An example of a well-resolved current sheet with an electron jet is shown in Figure 3. Panel (a) shows the magnetic field components, demonstrating a change in sign of $B_{L}$ (red) over approximately 1s, a guide field with bipolar Hall fields in $B_{M}$ (green), and a reduction in field magnitude (black). The field magnitude is not symmetric across the current sheet; it transitions from $40 \mathrm{nT}$ to $20 \mathrm{nT}$ over $3 \mathrm{~s}$, with an intermediate plateau for $1.5 \mathrm{~s}$ where $B_{L} \approx 0$. This is consistent with an asymmetric current sheet embedded within an inhomogeneous transition layer. However, we note that significant asymmetry is only visible within the magnetic fields. The electron and ion densities are symmetric, with $n_{e, i} \approx 70 \mathrm{~cm}^{3}$ throughout the interval. Under Taylor's hypothesis, using the normal component of the bulk velocity, this corresponds to a current sheet width of 3 ion inertial lengths.

Panel (b), showing bulk velocities, and panels (c)-(d) showing current densities, reveal that the current in $V_{M}$ (green) is carried by the electrons. The ion bulk velocities (dashed lines) do not vary across the current sheet. The reconnection jet is visible in $V e_{L}$ 

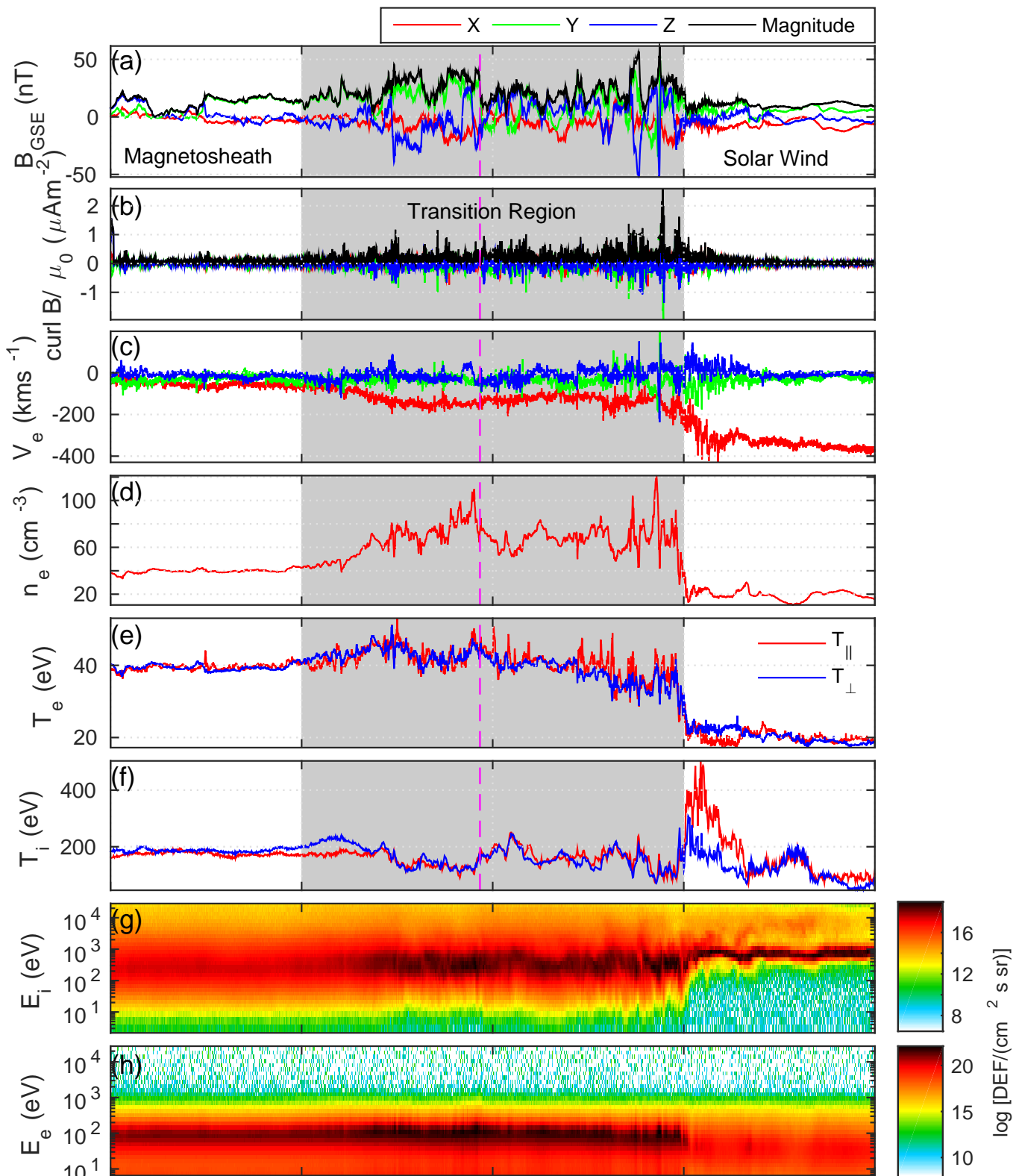
08:13:04
08:14:04
08:15:04
08:16:04
08:17:04

Time, UTC 26 Jan 2017

Figure 2. Overview of the bow shock crossing observed by MMS1 on 26th January 2017,

08:13:04 UTC, in Geocentric Solar Equatorial (GSE) coordinates. From top to bottom: magnetic field, curl of the magnetic field, electron bulk velocity, electron number density, electron temperature, ion temperature, spectrograms of the differential energy flux for ions and electrons. A disordered transition region is evident for the period 08:14:04 to 08:16:04 UTC, shown in grey. The dashed magenta lines show the time of the event in Figure 3. 
(red) as a deviation from the background velocity in the $-L$ direction, centered on the dashed vertical line. For a current sheet, a peak in the bulk velocity in the maximum variance $L$ direction is indicative of reconnection. Both the current and jet are associated with the high-field side of the separatrix, as expected for asymmetric reconnection (Eastwood et al., 2013). It is important to note that no jet is visible in the ion bulk velocity. The peak electron velocity at the centre of the jet is approximately $1.2 V_{A}$ for the mean Alfvén speed across the transition region, or $3.2 V_{A L \text {,inflow }}$, where $\left.V_{A L, \text { inflow }}=\left[B_{L, 1} B_{L, 2}\left(B_{L, 1}+B_{L, 2}\right) / \mu_{0}\left(\rho_{1} B_{L, 1}+\rho_{2} B_{L, 2}\right)\right]^{0.5}\right]$. Subscripts 1 and 2 denote the regions either side of the current carrying region (shown in Figure 3 with orange dashed lines) and $\rho$ is the ion mass density. Given the directions of the magnetic field, current and electron jet, we can infer the trajectory of spacecraft through an idealised reconnection site. This trajectory is shown with a red arrow in the top left of Figure 1. We note that all four MMS spacecraft observe similar features, suggesting all four cross the current sheet on the same side of the diffusion region.

The appearance of a reconnecting electron jet is further supported by the correlation between $V e_{L}$ and $B_{L}$. A scatter plot is shown inset in Figure 3. The jet is Alfvénic, lying principally along the Walen slopes $B_{L} \propto \pm V e_{L}\left(\mu_{0} \rho\right)^{1 / 2}$ (dashed lines), positively correlated approaching the electron jet (red points), and anti-correlated on passing the electron jet (blue points).

The electron jet is coincident with peaks in the perpendicular and parallel electron temperatures, corresponding to a $3 \mathrm{eV}$ increase. The mean electron temperature increase across the current-carrying region (shown with orange dashed lines in Figure 3 ) is $0.5 \mathrm{eV}$. However, as with the bulk velocities, ion temperatures do not show similar peaks. This further suggests that ions are not coupled to reconnection processes for this current sheet, despite the fact that the current sheet width is on the order of the ion inertial length. Another measure of heating, $\mathbf{J} \cdot \mathbf{E}^{\prime}$, where $\mathbf{E}^{\prime}=\mathbf{E}+\mathbf{v}_{e} \times \mathbf{B}$, is shown in panel (g). This corresponds to the exchange of energy between particles and fields in the particle rest frame. Such a feature may be visible for $0.5 \mathrm{~s}$ before the peak velocity of the electron jet. However, given the fluctuations of similar magnitude in the preceding second of the interval, it is unclear whether this feature is linked to ongoing heating driven by reconnection. 


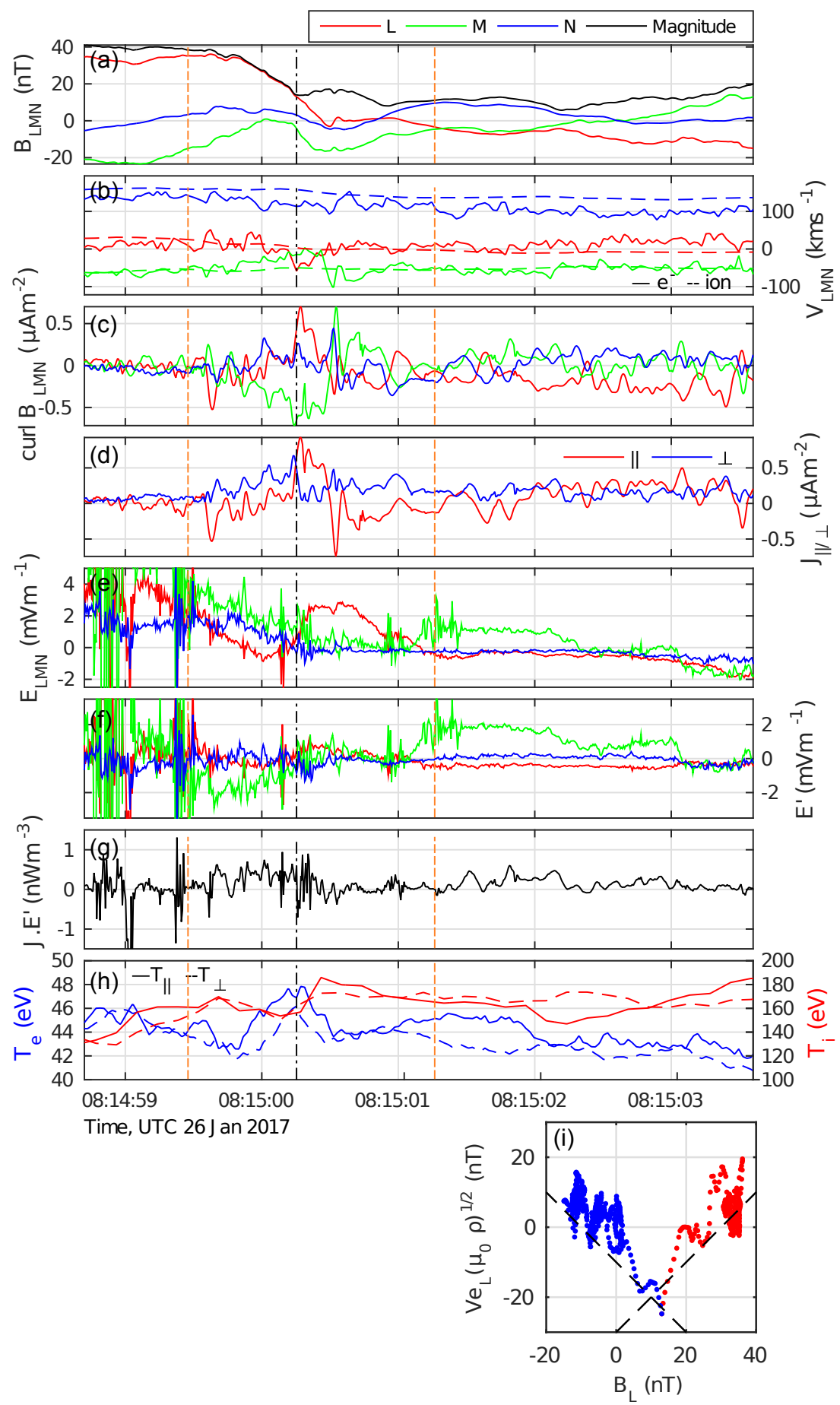

Figure 3. Observation of a reconnecting current sheet within the transition region, presented in a minimum variance coordinate system, and in the spacecraft frame. From top to bottom: magnetic field, electron (solid) and ion (dashed) bulk velocity, curl of the magnetic field, current density parallel and perpendicular to the magnetic field, electric fields, $\mathbf{E}^{\prime}=\mathbf{E}+\mathbf{V}_{e} \times \mathbf{B}$, heating measure $\mathbf{J} \cdot \mathbf{E}^{\prime}$, and electron and ion temperatures, and scatter showing correlation of the $L$-component of the electron bulk velocity and magnetic field. The dashed black vertical line is centered on the peak of the electron jet observed in $V e_{L}$, and the orange dashed vertical lines surround the current-carrying region. Data in thesscatter are coloured according to whether they are recorded before (red) or after (blue) crossing the electron jet. 
The preceding analysis demonstrates that reconnection occurs within the transition region of a quasi-parallel shock. Similarly to recent observations of magnetosheath reconnection (Phan et al., 2018), the outflow jet and particle heating appear limited to electrons. However, in this example, the current sheet width is larger; on the order of the ion inertial length. Given the lack of ion response, this suggests that this feature is relatively young, on the order of the ion gyro-period or less, and may have just formed. This is supported by recent hybrid simulations, which suggest that reformation-driven generation of current sheets occurs on timescales faster than the ion gyro-period (Gingell et al., 2017). It may be that an ion jet exists further from the reconnection site than the spacecraft trajectories pass. Although we do not observe clear ion jets for any other potential reconnection event associated with this shock, it may be that ion jets embedded within the turbulent structure of the transition region exhibit unexpected orientations.

\section{Conclusion}

Using observations of Earth's bow shock by MMS, we have demonstrated that reconnecting current sheets are present in the transition region of quasi-parallel shocks. Several reconnection jets have been observed within the shock shown in Figure 2, the clearest of which is shown in Figure 3. A further example of a current sheet, discovered within the transition region of another bow shock crossing observed by MMS on 31st December 2016, 06:06:24 UTC, is shown in the supporting information (Phan et al., 2014). The observation of current sheets is consistent with the magnetic structure of the transition region reported in hybrid simulations by Gingell et al. (2017). Magnetic reconnection may therefore play an important role in the energetics of collisionless shocks. However, given the hybrid nature of these simulations, they cannot accurately capture the observed electron-dominated plasma response.

Observations of the magnetopause suggest that $1.7 \%$ of the available inflow magnetic energy is transferred to the electrons during reconnection (Phan et al., 2013), i.e. $\Delta T_{e}=0.017 m_{i} V_{A L, \text { inflow }}$ where $\left.V_{A L, \text { inflow }}=\left[B_{L, 1} B_{L, 2}\left(B_{L, 1}+B_{L, 2}\right) / \mu_{0}\left(\rho_{1} B_{L, 1}+\rho_{2} B_{L, 2}\right)\right]^{0.5}\right]$. Subscripts 1 and 2 denote the regions either side of the current carrying region and $\rho$ is the ion mass density. For the asymmetric current sheet detailed in Figure 3, we take the fields and densities at the orange dashed lines such that $B_{L,[1,2]}=[35,4.0] n T$ and $n_{1,2}=70 \mathrm{~cm}^{-3}$. In this case, $V_{A L \text {,inflow }}=30 \mathrm{kms}^{-1}$ and hence $\Delta T_{e}=0.2 \mathrm{eV}$. This is 
consistent with a mean electron temperature increase of $0.5 \mathrm{eV}$ across the current sheet. However, we note that reconnection at the shock appears to partition energy differently to magnetopause reconnection, favouring the electrons. The total heating across the transition region can be seen in panels (e) and (f) of Figure 2. We find that the electron temperature rises from $20 \mathrm{eV}$ to $33 \mathrm{eV}$ in the ramp, and continues to rise another $7 \mathrm{eV}$ within the transition region. Thus, $35 \%$ of the total shock electron heating occurs in the transition region. We note that no similar trend is visible in the ion temperatures, suggesting again that dissipative processes in this region affect only electrons. We can estimate the ability of reconnection to provide the observed $7 \mathrm{eV}$ heating by considering the magnetic energy of the fluctuations per electron, $E_{f}=\left\langle(\delta B)^{2}\right\rangle /\left(2 \mu_{0} n_{e}\right)$, where $\delta B=|\mathbf{B}-\langle\mathbf{B}\rangle|$ and $\langle\mathbf{B}\rangle$ is the mean field across the transition region highlighted in Figure 2. For the transition region shown in Figure 2, $E_{f}=20 \mathrm{eV}$ per electron, while in the magnetosheath $E_{f}=10 \mathrm{eV} . \mathrm{A} 10 \mathrm{eV}$ dissipation is consistent with the observed $7 \mathrm{eV}$ electron temperature increase across the transition region. However, further work is required to establish the balance between reconnection and other dissipative processes in accounting for this temperature change.

Mechanisms for electron heating are strongly dependent on shock parameters such as the Mach number, $\theta_{B n}$, and plasma betas (Ghavamian, Schwartz, Mitchell, Masters, \& Laming, 2013). At supernova remnants, heating can be driven by waves excited by shock reflected ions or streaming cosmic rays, via the lower hybrid drift instability (Ghavamian, Laming, \& Rakowski, 2007) or the Buneman instability (for $M_{A}>50$ ) (Cargill \& Papadopoulos, 1988). Within the solar wind, heating may be driven by a modified two-stream instability or electron cyclotron drift instability (Matsukiyo, 2010; Umeda, Kidani, Matsukiyo, \& Yamazaki, 2012), or simply by the cross shock potential (Lefebvre, Schwartz, Fazakerley, \& Décréau, 2007). However, these mechanisms are most efficient for quasiperpendicular shocks. Thus, the observation of reconnection-driven heating at a quasiparallel shock represents an important development in the characterisation of energy partition at shocks in both astrophysical and space plasmas.

The reconnection event featured in this paper represents a regime in which the current and reconnection outflows are associated only with electrons, similar to the magnetosheath event reported by (Phan et al., 2018). However, in this case the scale lengths are on the order of the ion inertial scale. No similar structures are observed at electron scales. Thus, the observed current sheets may represent the end-stage of a turbulent cas- 
cade which dissipates energy at ion scales. The observed lack of ion response may alternatively indicate a rapid onset time. Given the proximity of some current sheets to the shock ramp, and the timescale for cyclic reformation for similar shocks (Gingell et al., 2017), the observed reconnection site may be younger than an ion gyro-period. In simulations (Gingell et al., 2017), rapid onset reconnection is driven at ion scales by instabilities in the foreshock and foot, generating coherent magnetic islands in the transition region. These instabilities, modulated by cyclic reformation, may generate a range of scales simultaneously, rather than by ongoing cascade as expected in magnetosheath turbulence. These structures then coalesce via secondary reconnection as they convect downstream, relaxing the magnetic field.

These observations support the need for more detailed simulations of reconnection at shocks, and observational surveys across all parameter regimes. This will allow us to asses the broader impact of reconnection on heating and particle acceleration at collisionless shocks, explore the evolution of these structures as they convect downstream, and determine how reconnection properties at coherent, rapidly-driven thin boundaries differ from models of reconnection operating elsewhere in the magnetosphere and heliosphere.

\section{Acknowledgments}

This work was supported by the UK Science and Technology Facilities Council (STFC) grant ST/N000692/1. Data used in this research is publicly available at the MMS Science Data Center at the Laboratory for Atmospheric and Space Physics (LASP) hosted by the University of Colorado, Boulder (https://lasp.colorado.edu/mms/sdc/public/). Work at IRAP is supported by CNRS and CNES.

\section{References}

Abraham-Shrauner, B. (1972). Determination of magnetohydrodynamic shock normals. Journal of Geophysical Research, 77, 736. doi: 10.1029/ JA077i004p00736

Auer, P. L., Hurwitz, H., Jr., \& Kilb, R. W. (1962, March). ～Large-Amplitude Magnetic Compression of a Collision-Free Plasma. II. Development of a Thermalized Plasma. Physics of Fluids, 5, 298-316. doi: 10.1063/1.1706615

Biskamp, D., \& Welter, H. (1972). Structure of the Earth's bow shock. Journal of 
Geophysical Research, 77, 6052. doi: 10.1029/JA077i031p06052

Bohdan, A., Niemiec, J., Kobzar, O., \& Pohl, M. (2017, September). Electron Preacceleration at Nonrelativistic High-Mach-number Perpendicular Shocks. The Astrophysical Journal, 847, 71. doi: 10.3847/1538-4357/aa872a

Burch, J. L., Torbert, R. B., Phan, T. D., Chen, L.-J., Moore, T. E., Ergun, R. E., ... Chandler, M. (2016, June). Electron-scale measurements of magnetic reconnection in space. Science, 352, aaf2939. doi: 10.1126/science.aaf2939

Burgess, D. (1989, May). Cyclic behavior at quasi-parallel collisionless shocks. Geophysical Research Letters, 16, 345-348. doi: 10.1029/GL016i005p00345

Burgess, D. (1995). Foreshock-shock interaction at collisionless quasi-parallel shocks. Advances in Space Research, 15, 159-169. doi: 10.1016/0273-1177(94)00098-L

Burgess, D., \& Scholer, M. (2015). Collisionless Shocks in Space Plasmas. Cambridge University Press.

Cargill, P. J., \& Papadopoulos, K. (1988, June). A mechanism for strong shock electron heating in supernova remnants. Astrophysical Journal Letters, 329, L29L32. doi: $10.1086 / 185170$

Eastwood, J. P., Phan, T. D., Øieroset, M., Shay, M. A., Malakit, K., Swisdak, M., ... Masters, A. (2013, December). Influence of asymmetries and guide fields on the magnetic reconnection diffusion region in collisionless space plasmas. $\quad$ Plasma Physics and Controlled Fusion, 55(12), $124001 . \quad$ doi: $10.1088 / 0741-3335 / 55 / 12 / 124001$

Ghavamian, P., Laming, J. M., \& Rakowski, C. E. （2007, January). A Physical Relationship between Electron-Proton Temperature Equilibration and Mach Number in Fast Collisionless Shocks. Astrophysical Journal Letters, 654, L69-L72. doi: 10.1086/510740

Ghavamian, P., Schwartz, S. J., Mitchell, J., Masters, A., \& Laming, J. M. $(2013$, October). Electron-Ion Temperature Equilibration in Collisionless Shocks: The Supernova Remnant-Solar Wind Connection. Space Science Reviews, 178, 633-663. doi: 10.1007/s11214-013-9999-0

Gingell, I., Schwartz, S. J., Burgess, D., Johlander, A., Russell, C. T., Burch, J. L., ... Wilder, F. (2017, November). MMS Observations and Hybrid Simulations of Surface Ripples at a Marginally Quasi-Parallel Shock. Journal of Geophysical Research (Space Physics), 122, 11. doi: 10.1002/2017JA024538 
Gosling, J. T., \& Phan, T. D. (2013, February). Magnetic Reconnection in the Solar Wind at Current Sheets Associated with Extremely Small Field Shear Angles. Astrophysical Journal Letters, 763, L39. doi: 10.1088/2041-8205/763/2/L39

Gosling, J. T., \& Robson, A. E. (1985). Ion reflection, gyration, and dissipation at supercritical shocks. Washington DC American Geophysical Union Geophysical Monograph Series, 35, 141-152. doi: 10.1029/GM035p0141

Gosling, J. T., Skoug, R. M., McComas, D. J., \& Smith, C. W.

(2005, January). Direct evidence for magnetic reconnection in the solar wind near 1 AU. Journal of Geophysical Research (Space Physics), 110, A01107. doi: 10.1029/2004JA010809

Hada, T., Oonishi, M., Lembège, B., \& Savoini, P. (2003, June). Shock front nonstationarity of supercritical perpendicular shocks. Journal of Geophysical Research (Space Physics), 108, 1233. doi: 10.1029/2002JA009339

Karimabadi, H., Roytershteyn, V., Vu, H. X., Omelchenko, Y. A., Scudder, J., Daughton, W., ... Geveci, B. (2014, June). The link between shocks, turbulence, and magnetic reconnection in collisionless plasmas. Physics of Plasmas, 21(6), 062308. doi: 10.1063/1.4882875

Krauss-Varban, D., \& Omidi, N. (1991, October). Structure of medium Mach number quasi-parallel shocks - Upstream and downstream waves. Journal of Geophysical Research, 96, 17. doi: 10.1029/91JA01545

Lefebvre, B., Schwartz, S. J., Fazakerley, A. F., \& Décréau, P. ～(2007, September). Electron dynamics and cross-shock potential at the quasi-perpendicular Earth's bow shock. Journal of Geophysical Research (Space Physics), 112, A09212. doi: 10.1029/2007JA012277

Matsukiyo, S. (2010, April). Mach number dependence of electron heating in high Mach number quasiperpendicular shocks. Physics of Plasmas, 17(4), 042901042901. doi: 10.1063/1.3372137

Matsumoto, Y., Amano, T., Kato, T. N., \& Hoshino, M. (2015). Stochastic electron acceleration during spontaneous turbulent reconnection in a strong shock wave. Science, 347(6225), 974-978. Retrieved from http://science.sciencemag .org/content/347/6225/974 doi: 10.1126/science.1260168

Matthaeus, W. H., \& Lamkin, S. L. (1986). Turbulent magnetic reconnection. The Physics of Fluids, 29(8), 2513-2534. Retrieved from https://aip.scitation 
.org/doi/abs/10.1063/1.866004 doi: 10.1063/1.866004

Morse, D. L., Destler, W. W., \& Auer, P. L. (1972, January). Nonstationary Behavior of Collisionless Shocks. Physical Review Letters, 28, 13-16. doi: 10.1103/ PhysRevLett.28.13

Paschmann, G., Papamastorakis, I., Sckopke, N., Haerendel, G., Sonnerup, B. U. O., Bame, S. J., .. Elphic, R. C. (1979, November). Plasma acceleration at the earth's magnetopause - Evidence for reconnection. Nature, 282, 243-246. doi: $10.1038 / 282243 \mathrm{a} 0$

Phan, T. D., Drake, J. F., Shay, M. A., Gosling, J. T., Paschmann, G., Eastwood, J. P., ... Angelopoulos, V. (2014, October). Ion bulk heating in magnetic reconnection exhausts at Earth's magnetopause: Dependence on the inflow Alfvén speed and magnetic shear angle. Geophys. Res. Lett., 41, 7002-7010. doi: 10.1002/2014GL061547

Phan, T. D., Eastwood, J. P., Shay, M. A., Drake, J. F., Sonnerup, B. U. Ö., Fujimoto, M., ... Magnes, W. (2018, May). Electron magnetic reconnection without ion coupling in Earth's turbulent magnetosheath. $\quad$ Nature, 557, 202206. doi: 10.1038/s41586-018-0091-5

Phan, T. D., Kistler, L. M., Klecker, B., Haerendel, G., Paschmann, G., Sonnerup, B. U. Ö., ... Reme, H. (2000, April). Extended magnetic reconnection at the Earth's magnetopause from detection of bi-directional jets. Nature, 404 , 848-850. doi: 10.1038/35009050

Phan, T. D., Shay, M. A., Gosling, J. T., Fujimoto, M., Drake, J. F., Paschmann, G., ... Angelopoulos, V. (2013, September). Electron bulk heating in magnetic reconnection at Earth's magnetopause: Dependence on the inflow Alfvén speed and magnetic shear. Geophysical Research Letters, 40, 4475-4480. doi: $10.1002 / \operatorname{grl} .50917$

Pollock, C., Moore, T., Jacques, A., Burch, J., Gliese, U., Saito, Y., ... Zeuch, M. (2016, March). Fast Plasma Investigation for Magnetospheric Multiscale. Space Science Reviews, 199, 331-406. doi: 10.1007/s11214-016-0245-4

Retinò, A., Sundkvist, D., Vaivads, A., Mozer, F., André, M., \& Owen, C. J. (2007, April). In situ evidence of magnetic reconnection in turbulent plasma. Nature Physics, 3, 236-238. doi: 10.1038/nphys574

Robert, P., Dunlop, M. W., Roux, A., \& Chanteur, G. (1998). Accuracy of Current 
Density Determination. ISSI Scientific Reports Series, 1, 395-418.

Russell, C. T., Anderson, B. J., Baumjohann, W., Bromund, K. R., Dearborn, D., Fischer, D., ... Richter, I. (2016, March). The Magnetospheric Multiscale Magnetometers. $\quad$ Space Science Reviews, 199, 189-256. doi: $10.1007 / \mathrm{s} 11214-014-0057-3$

Scholer, M., \& Burgess, D. (2007, July). Whistler waves, core ion heating, and nonstationarity in oblique collisionless shocks. Physics of Plasmas, 14 (7), 072103. doi: $10.1063 / 1.2748391$

Scholer, M., Shinohara, I., \& Matsukiyo, S. (2003, January). Quasi-perpendicular shocks: Length scale of the cross-shock potential, shock reformation, and implication for shock surfing. Journal of Geophysical Research (Space Physics), 108, 1014. doi: 10.1029/2002JA009515

Schwartz, S. J. (1998). Analysis Methods for Multi-Spacecraft Data. ISSI Sci. Rep. Ser., 1, 249.

Servidio, S., Matthaeus, W. H., Shay, M. A., Cassak, P. A., \& Dmitruk, P. (2009, March). Magnetic Reconnection in Two-Dimensional Magnetohydrodynamic Turbulence. $\quad$ Physical Review Letters, 102(11), $115003 . \quad$ doi: 10.1103/PhysRevLett.102.115003

Sundkvist, D., Retinò, A., Vaivads, A., \& Bale, S. D. (2007, July). Dissipation in Turbulent Plasma due to Reconnection in Thin Current Sheets. Physical Review Letters, 99(2), 025004. doi: 10.1103/PhysRevLett.99.025004

Torbert, R. B., Russell, C. T., Magnes, W., Ergun, R. E., Lindqvist, P.-A., LeContel, O., ... Lappalainen, K. (2016, March). The FIELDS Instrument Suite on MMS: Scientific Objectives, Measurements, and Data Products. Space Science Reviews, 199, 105-135. doi: 10.1007/s11214-014-0109-8

Umeda, T., Kidani, Y., Matsukiyo, S., \& Yamazaki, R. (2012, March). Modified two-stream instability at perpendicular collisionless shocks: Full particle simulations. Journal of Geophysical Research (Space Physics), 117, A03206. doi: 10.1029/2011JA017182

Vasyliunas, V. M. (1975, February). Theoretical models of magnetic field line merging. I. Reviews of Geophysics and Space Physics, 13, 303-336. doi: 10.1029/ RG013i001p00303 